Ioannis K. Argyros · Santhosh George

\title{
Ball convergence theorems for eighth-order variants of Newton's method under weak conditions
}

Received: 12 November 2014 / Accepted: 24 March 2015 / Published online: 10 April 2015

(C) The Author(s) 2015. This article is published with open access at Springerlink.com

\begin{abstract}
We present a local convergence analysis for eighth-order variants of Newton's method in order to approximate a solution of a nonlinear equation. We use hypotheses up to the first derivative in contrast to earlier studies such as Amat et al. (Appl Math Comput 206(1):164-174, 2008), Amat et al. (Aequationes Math 69:212-213, 2005), Chun et al. (Appl Math Comput. 227:567-592, 2014), Petkovic et al. (Multipoint methods for solving nonlinear equations. Elsevier, Amsterdam, 2013), Potra and Ptak (Nondiscrete induction and iterative processes. Pitman Publ, Boston, 1984), Rall (Computational solution of nonlinear operator equations. Robert E. Krieger, New York, 1979), Ren et al. (Numer Algorithms 52(4):585-603, 2009), Rheinboldt (An adaptive continuation process for solving systems of nonlinear equations. Banach Center, Warsaw, 1975), Traub (Iterative methods for the solution of equations. Prentice Hall, Englewood Cliffs, 1964), Weerakoon and Fernando (Appl Math Lett 13:87-93, 2000), Wang and Kou (J Differ Equ Appl 19(9):1483-1500, 2013) using hypotheses up to the seventh derivative. This way the applicability of these methods is extended under weaker hypotheses. Moreover, the radius of convergence and computable error bounds on the distances involved are also given in this study. Numerical examples are also presented in this study.
\end{abstract}

Mathematics Subject Classification 65D10 - 65D99

نقام تحليل تقارب محلي لأشكال من الارجة الثامنة لطريقة نيوتن لتقريب حل لمعادلة غير خطية. نستخدم فرضيات حتى المشتقة الأولى للتفرقة بين هذه

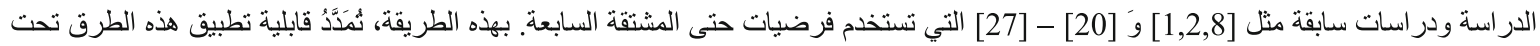

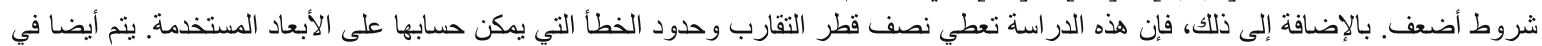

هذه الدر اسة عرض أمثلة عددية.

\section{Introduction}

In this study, we are concerned with the problem of approximating a locally unique solution $x^{*}$ of equation

$$
F(x)=0,
$$

where $F: D \subseteq S \rightarrow S$ is a nonlinear function, $D$ is a convex subset of $S$ and $S$ is $\mathbb{R}$ or $\mathbb{C}$. Newton-like methods are used for finding solution of (1.1), these methods are usually studied based on semi-local and local

I. K. Argyros

Department of Mathematical Sciences, Cameron University, Lawton, OK 73505, USA

E-mail: iargyros@ cameron.edu

S. George $(\varangle)$

Department of Mathematical and Computational Sciences, NIT Karnataka, Mangalore 575 025, India

E-mail: sgeorge@nitk.ac.in 
convergence. The semi-local convergence matter is, based on the information around an initial point, to give conditions ensuring the convergence of the iterative procedure; while the local one is, based on the information around a solution, to find estimates of the radii of convergence balls [3,4,20-22,24,25].

Third-order methods such as Euler's, Halley's, super Halley's, Chebyshev's [1-27] require the evaluation of the second derivative $F^{\prime \prime}$ at each step, which in general is very expensive. That is why many authors have used higher order multipoint methods [1-27]. In this paper, we study the local convergence of eighth-order method defined for each $n=0,1,2, \ldots$ by

$$
\begin{aligned}
y_{n} & =x_{n}-\frac{2}{3} F^{\prime}\left(x_{n}\right)^{-1} F\left(x_{n}\right) \\
z_{n} & =x_{n}-\frac{1}{2} F^{\prime}\left(x_{n}\right)^{-1} F\left(x_{n}\right)-\frac{3}{2}\left[1+2 F^{\prime}\left(x_{n}\right)^{-1} F^{\prime}\left(y_{n}\right)\right]^{-1} F^{\prime}\left(x_{n}\right)^{-1} F\left(x_{n}\right) \\
x_{n+1} & =z_{n}-A_{n}^{-1} F\left(z_{n}\right),
\end{aligned}
$$

where $x_{0}$ is an initial point,

$$
A_{n}=2\left(\left[x_{n}, z_{n} ; F\right]-\left[x_{n}, y_{n} ; F\right]\right)+\left[y_{n}, z_{n} ; F\right]+\frac{y_{n}-z_{n}}{y_{n}-x_{n}}\left(\left[x_{n}, y_{n} ; F\right]-F^{\prime}\left(x_{n}\right)\right),
$$

$[x, y ; F]=\frac{F(y)-F(x)}{y-x}, y \neq x$ with $F^{\prime}(x)=[x, x ; F]$ is a divided difference of order one for function $F$ at the point $x$ and $y[3,4,20-22,25]$. Petkovic et al. [20] developed several eighth-order methods using optimal fourth-order methods followed by a step of interpolation. In particular they showed using Taylor expansions that the order of convergence for method (1.2) is eight. Method (1.2) has already been shown in [8] (see also [20]) to be performing better than other competing methods using similar information. Several authors have also studied eight order methods [2,8-11] or methods of order higher than 2 [1,3-7,12-27].

Other single and multipoint methods can be found in $[2,3,20,25]$ and the references therein. The local convergence of the preceding methods has been shown under hypotheses up to the seventh derivative (or even higher) although only the first derivative appears in method (1.2). These hypotheses restrict the applicability of these methods. As a motivational example, let us define function $f$ on $D=\left[-\frac{1}{2}, \frac{5}{2}\right]$ by

$$
f(x)=\left\{\begin{array}{l}
2 x^{3} \ln x+x^{5}-x^{4}, \quad x \neq 0 \\
0, \quad x=0
\end{array}\right.
$$

Choose $x^{*}=1$. We have that

$$
\begin{aligned}
& f^{\prime}(x)=6 x^{2} \ln x+5 x^{4}-4 x^{3}+2 x^{2}, f^{\prime}(1)=3, \\
& f^{\prime \prime}(x)=12 x \ln x+20 x^{3}-12 x^{2}+10 x \\
& f^{\prime \prime \prime}(x)=12 \ln x+60 x^{2}-24 x+22 .
\end{aligned}
$$

Then, obviously, function $f^{\prime \prime \prime}$ is unbounded on $D$. In the present paper, we use hypotheses only on the first derivative. Moreover, we provide a radius of convergence and computable error estimates on the distances using $\left|x_{n}-x^{*}\right|$ with Lipschitz constants not provided in the earlier studies by Taylor expansions. This way we expand the applicability of method (1.2).

The rest of the paper is organized as follows: Sect. 2 contains the local convergence analysis of methods (1.2). The numerical examples are presented in the concluding Sect. 3.

\section{Local convergence for method (1.2)}

We present the local convergence analysis of method (1.2) in this section. Let $U(v, \rho), \bar{U}(v, \rho)$ stand for the open and closed balls in $S$, respectively, with center $v \in S$ and of radius $\rho>0$.

For the local convergence analysis that follows we define some functions and parameters. Let $L_{i}>0, i=$ $0,1,2,3,4, M \in(0,3)$ be given parameters. Define functions on the interval $\left[0, \frac{1}{L_{0}}\right)$ by

$$
g_{1}(t)=\frac{1}{2\left(1-L_{0} t\right)}\left(L t+\frac{2 M}{3}\right),
$$


and parameters

$$
r_{1}=\frac{2\left(1-\frac{M}{3}\right)}{2 L_{0}+L}<r_{A}=\frac{2}{2 L_{0}+L}<\frac{1}{L_{0}} .
$$

Notice that $r_{1}>0$ and $g_{1}\left(r_{1}\right)=1$. Moreover, define functions on the interval $\left[0, \frac{1}{L_{0}}\right)$ by

$$
g_{0}(t)=\frac{L_{0}}{2}\left(5+3 g_{1}(t)\right) t
$$

and

$$
h_{0}(t)=g_{0}(t) t-1 \text {. }
$$

We have that $h_{0}(0)=-1<0$ and $h_{0}(t) \rightarrow+\infty$ as $t \rightarrow{\frac{1}{L_{0}}}^{-}$. It follows from the intermediate value theorem that function $h_{0}$ has zeros in the interval $\left(0, \frac{1}{L_{0}}\right)$. Denote by $r_{0}$ the smallest such zero. Then, define functions on the interval $\left[0, r_{0}\right)$ by

$$
g_{2}(t)=\frac{1}{2\left(1-L_{0} t\right)}\left[L+\frac{3 M L_{0}\left(1+g_{1}(t)\right)}{2\left(1-g_{0}(t)\right)}\right] t
$$

and

$$
h_{2}(t)=g_{2}(t)-1 .
$$

We have that $h_{2}(0)=-1<0$ and $h_{2}(t) \rightarrow+\infty$ as $t \rightarrow r_{0}^{-}$. Hence, function $h_{2}$ has zeros in the interval $\left(0, r_{0}\right)$. Denote the smallest such zero by $r_{2}$. Furthermore, define functions $p, p_{1}$ and $p_{2}$ on the interval $\left(0, r_{0}\right)$ by

$$
\begin{gathered}
p(t)=\frac{1}{4} \frac{2\left(1+L_{0} t\right)+\frac{3}{2} L_{0}\left(1+g_{1}(t)\right) t}{1-g_{0}(t)} \\
p_{1}(t)=\left[\left(5 L_{1}+p(t)\left(L_{0}+L_{1}\right)\right)+(2+p(t)) L_{2} g_{1}(t)+3 L_{2} g_{2}(t)\right] t
\end{gathered}
$$

and

$$
p_{2}(t)=\left[\left(L_{1}+L_{4} p(t)\right)+\left(2 L_{3}+L_{4} p(t)\right) g_{1}(t)+\left(L_{2}+2 L_{3}\right) g_{2}(t)\right] t
$$

set $\bar{p}_{1}(t)=p_{1}(t)-1$ and $\overline{p_{2}}=p_{2}(t)-1$. We have that $\bar{p}_{1}(0)=-1<0$ and $\overline{p_{1}}(t) \rightarrow+\infty$ as $t \rightarrow r_{0}^{-}$. Hence, function $\bar{p}_{1}$ has a smallest zero in $\left(0, r_{0}\right)$ denoted by $r_{\overline{p_{1}}}$. Similarly function $\overline{p_{2}}$ has a smallest zero in $\left(0, r_{0}\right)$ denoted by $r_{\overline{p_{2}}}$. Define function

$$
p_{0}= \begin{cases}p_{1}, & \text { if } \quad r_{\overline{p_{2}}} \leq r_{\overline{p_{1}}} \\ p_{2}, & \text { if } \quad r_{\overline{p_{1}}} \leq r_{\overline{p_{2}}}\end{cases}
$$

Finally, define function on the interval $\left[0, r_{0}\right)$ by

$$
g_{3}(t)=\left[1+\frac{M}{1-p_{0}(t)}\right] g_{2}(t)
$$

and set $h_{3}(t)=g_{3}(t)-1$. We have that $h_{3}(0)=-1$ and $h_{3}(t) \rightarrow+\infty$ as $t \rightarrow r_{\overline{p_{1}}}$ if $p_{0}=p_{1}$ or as $t \rightarrow r_{\overline{p_{2}}}$ if $p_{0}=p_{2}$. Hence, function $h_{3}$ has a smallest zero denoted by $r_{3}$ in the interval $\left(0, r_{0}\right)$. Set

$$
r=\min \left\{r_{1}, r_{2}, r_{3}, r_{\overline{p_{1}}}, r_{\overline{p_{2}}}\right\} .
$$

Then, we have that

$$
\begin{aligned}
& 0 \leq g_{1}(t)<1, \\
& 0 \leq g_{0}(t)<1, \\
& 0 \leq g_{2}(t)<1, \\
& 0 \leq p_{0}(t)<1,
\end{aligned}
$$

and 


$$
0 \leq g_{3}(t)<1 \text { for each } t \in[0, r) .
$$

Function $p_{0}$ is defined in terms of $L_{0}, L_{1}, L_{2}, g_{0}, g_{1}$ and $g_{2}$ (i.e., as function of $p_{1}$ ) or in terms of $L_{0}, L_{1}, L_{4}, g_{0}, g_{1}$ and $g_{2}$ (i.e., as function of $p_{2}$ ). In practice, we shall choose the choice of $p_{0}$ leading to the largest radius which will be $r_{\overline{p_{1}}}$ or $r_{\overline{p_{2}}}$, since we need to obtain the largest possible convergence ball.

Next, using the above notation we can present the local convergence analysis of method (1.2).

Theorem 2.1 Let $F: D \subseteq S \rightarrow S$ be a differentiable function. Suppose that there exist a divided difference of order one $[., . ; F]: D \times D \rightarrow L(S, S), x^{*} \in D$, parameters $L_{i}>0, i=1,2, \ldots, 4, M \in(0,3)$ such that for each $x, y \in D$ the following hold

$$
\begin{gathered}
F\left(x^{*}\right)=0, F^{\prime}\left(x^{*}\right) \neq 0, \\
\left|F^{\prime}\left(x^{*}\right)^{-1}\left(F^{\prime}(x)-F^{\prime}\left(x^{*}\right)\right)\right| \leq L_{0}\left|x-x^{*}\right|, \\
\left|F^{\prime}\left(x^{*}\right)^{-1}\left(F^{\prime}(x)-F^{\prime}(y)\right)\right| \leq L|x-y|, \\
\left|F^{\prime}\left(x^{*}\right)^{-1}\left([x, y ; F]-F^{\prime}\left(x^{*}\right)\right)\right| \leq L_{1}\left|x-x^{*}\right|+L_{2}\left|y-x^{*}\right|, \\
\left|F^{\prime}\left(x^{*}\right)^{-1}([x, z ; F]-[x, y ; F])\right| \leq L_{3}|z-y|, \\
\left|F^{\prime}\left(x^{*}\right)^{-1}\left([x, y ; F]-F^{\prime}(x)\right)\right| \leq L_{4}|x-y|, \\
\left|F^{\prime}\left(x^{*}\right)^{-1} F^{\prime}(x)\right| \leq M
\end{gathered}
$$

and

$$
\bar{U}\left(x^{*}, r\right) \subseteq D,
$$

where $r$ is defined above Theorem 2.1. Then, sequence $\left\{x_{n}\right\}$ generated for $x_{0} \in U\left(x^{*}, r\right)$ by method $(1.2)$ is well defined, remains in $U\left(x^{*}, r\right)$ for each $n=0,1,2, \ldots$ and converges to $x^{*}$. Moreover, the following estimates hold for each $n=0,1,2, \ldots$,

$$
\begin{gathered}
\left|y_{n}-x^{*}\right| \leq g_{1}\left(\left|x_{n}-x^{*}\right|\right)\left|x_{n}-x^{*}\right|<\left|x_{n}-x^{*}\right|<r, \\
\left|z_{n}-x^{*}\right| \leq g_{2}\left(\left|x_{n}-x^{*}\right|\right)\left|x_{n}-x^{*}\right|<\left|x_{n}-x^{*}\right|
\end{gathered}
$$

and

$$
\left|x_{n+1}-x^{*}\right| \leq g_{3}\left(\left|x_{n}-x^{*}\right|\right)\left|x_{n}-x^{*}\right|<\left|x_{n}-x^{*}\right|,
$$

where the " $g$ " functions are defined above Theorem 2.1. Furthermore, suppose that there exists $T \in\left[r, \frac{2}{L_{0}}\right)$ such that $\bar{U}\left(x^{*}, T\right) \subset D$. Then the limit point $x^{*}$ is the only solution of equation $F(x)=0$ in $\bar{U}\left(x^{*}, T\right)$.

Proof By hypothesis $x_{0} \in U\left(x^{*}, r\right)$, the definition of $r$ and (2.9) we get that

$$
\left|F^{\prime}\left(x^{*}\right)^{-1}\left(F^{\prime}\left(x_{0}\right)-F^{\prime}\left(x^{*}\right)\right)\right| \leq L_{0}\left|x_{0}-x^{*}\right|<L_{0} r<1 .
$$

It follows from (2.19) and the Banach Lemma on invertible functions $[3,4,19,20,22,23]$ that $F^{\prime}\left(x_{0}\right)$ is invertible and

$$
\left|F^{\prime}\left(x_{0}\right)^{-1} F^{\prime}\left(x^{*}\right)\right| \leq \frac{1}{1-L_{0}\left|x_{0}-x^{*}\right|}<\frac{1}{1-L_{0} r}
$$


Hence, $y_{0}$ is well defined by the first substep of method (1.2) for $n=0$. We also have that

$$
\begin{aligned}
y_{0}-x^{*}= & x_{0}-x^{*}-\frac{F\left(x_{0}\right)}{F^{\prime}\left(x_{0}\right)}+\frac{1}{3} \frac{F\left(x_{0}\right)}{F^{\prime}\left(x_{0}\right)} \\
= & -\left[F^{\prime}\left(x_{0}\right)^{-1} F^{\prime}\left(x^{*}\right)\right]\left[\int_{0}^{1} F^{\prime}\left(x^{*}\right)^{-1}\right. \\
& \left.\times\left[F\left(x^{*}+\theta\left(x_{0}-x^{*}\right)\right)-F^{\prime}\left(x_{0}\right)\right]\left(x_{0}-x^{*}\right) d \theta\right] \\
& +\frac{1}{3}\left[F^{\prime}\left(x_{0}\right)^{-1} F^{\prime}\left(x^{*}\right)\right]\left[\int_{0}^{1} F^{\prime}\left(x^{*}\right)^{-1}\right. \\
& \times\left[F\left(x^{*}+\theta\left(x_{0}-x^{*}\right)\right)\left(x_{0}-x^{*}\right) d \theta\right] .
\end{aligned}
$$

Using (2.2), (2.3), (2.10), (2.14) and (2.21) we get in turn that

$$
\begin{aligned}
\left|y_{0}-x^{*}\right| \leq & \left|F^{\prime}\left(x_{0}\right)^{-1} F^{\prime}\left(x^{*}\right)\right| \\
& \times \mid \int_{0}^{1} F^{\prime}\left(x^{*}\right)^{-1}\left[F^{\prime}\left(x^{*}+\theta\left(x_{0}-x^{*}\right)-F^{\prime}\left(x_{0}\right)\right] d \theta|| x_{0}-x^{*} \mid\right. \\
& +\left|F^{\prime}\left(x_{0}\right)^{-1} F^{\prime}\left(x^{*}\right)\right| \\
& \times \mid \int_{0}^{1} F^{\prime}\left(x^{*}\right)^{-1} F^{\prime}\left(x^{*}+\theta\left(x_{0}-x^{*}\right) d \theta|| x_{0}-x^{*} \mid\right. \\
\leq & \frac{L\left|x_{0}-x^{*}\right|^{2}}{2\left(1-L_{0}\left|x_{0}-x^{*}\right|\right)}+\frac{M\left|x_{0}-x^{*}\right|}{3\left(1-L_{0}\left|x_{0}-x^{*}\right|\right)} \\
= & g_{1}\left(\left|x_{0}-x^{*}\right|\right)\left|x_{0}-x^{*}\right|<\left|x_{0}-x^{*}\right|<r,
\end{aligned}
$$

which shows (2.16) for $n=0$, where we used $\left|x^{*}+\theta\left(x_{0}-x^{*}\right)\right|=\theta\left|x_{0}-x^{*}\right| \leq\left|x_{0}-x^{*}\right|<r$, that is $x^{*}+\theta\left(x_{0}-x^{*}\right) \in U\left(x^{*}, r\right)$ for each $\theta \in[0,1]$. Next, we shall show that $B_{0}$ is invertible, where

$$
B_{0}=F^{\prime}\left(x_{0}\right)+\frac{3}{2}\left(F^{\prime}\left(y_{0}\right)-F^{\prime}\left(x_{0}\right)\right) .
$$

Using (2.2), (2.3), (2.4), (2.16) and (2.22) we get

$$
\begin{aligned}
\mid F^{\prime}\left(x^{*}\right)^{-1}\left(B_{0}-F^{\prime}\left(x^{*}\right) \mid \leq\right. & \left|F^{\prime}\left(x^{*}\right)^{-1}\left(F^{\prime}\left(x_{0}\right)-F^{\prime}\left(x^{*}\right)\right)\right| \\
& +\frac{3}{2}\left|F^{\prime}\left(x^{*}\right)^{-1}\left(F^{\prime}\left(y_{0}\right)-F^{\prime}\left(x^{*}\right)\right)\right| \\
& +\left|F^{\prime}\left(x^{*}\right)^{-1}\left(F^{\prime}\left(x_{0}\right)-F^{\prime}\left(x^{*}\right)\right)\right| \\
\leq & L_{0}\left[\left|x_{0}-x^{*}\right|+\frac{3}{2}\left(\left|y_{0}-x^{*}\right|+\left|x_{0}-x^{*}\right|\right)\right] \\
\leq & L_{0}\left[1+\frac{3}{2}\left(g_{1}\left(\left|x_{0}-x^{*}\right|\right)+1\right)\right]\left|x_{0}-x^{*}\right| \\
= & g_{0}\left(\left|x_{0}-x^{*}\right|\right)<g_{0}(r)<1 .
\end{aligned}
$$

It follows from (2.23) that $B_{0}$ is invertible and

$$
\left|B_{0}^{-1} F^{\prime}\left(x^{*}\right)\right| \leq \frac{1}{1-g_{0}\left(\left|x_{0}-x^{*}\right|\right)} .
$$

Hence, $z_{0}$ is well defined by the second substep of method (1.2) for $n=0$. Then, we can write

$$
\begin{aligned}
z_{0}-x^{*}= & {\left[x_{0}-x^{*}-F^{\prime}\left(x_{0}\right)^{-1} F\left(x_{0}\right)\right] } \\
& +\frac{1}{2} F^{\prime}\left(x_{0}\right)^{-1} F\left(x_{0}\right)\left[1-\frac{1}{1+\frac{3}{2}\left(\frac{F^{\prime}\left(y_{0}\right)-F^{\prime}\left(x_{0}\right)}{F^{\prime}\left(x_{0}\right)}\right)}\right]
\end{aligned}
$$




$$
\begin{aligned}
= & {\left[x_{0}-x^{*}-F^{\prime}\left(x_{0}\right)^{-1} F\left(x_{0}\right)\right] } \\
& +\frac{3}{4} F^{\prime}\left(x_{0}\right)^{-1} F\left(x_{0}\right) B_{0}^{-1}\left(F^{\prime}\left(y_{0}\right)-F^{\prime}\left(x_{0}\right)\right) .
\end{aligned}
$$

Then, we have by (2.2), (2.3), (2.4), (2.5), (2.9), (2.14), (2.16), (2.24) and (2.25) that

$$
\begin{aligned}
\left|z_{0}-x^{*}\right| \leq & \left|x_{0}-x^{*}-F^{\prime}\left(x_{0}\right)^{-1} F\left(x_{0}\right)\right|+\frac{3}{4}\left|F^{\prime}\left(x_{0}\right)^{-1} F\left(x_{0}\right)\right|\left|B_{0}^{-1} F^{\prime}\left(x^{*}\right)\right| \\
& \times\left|F^{\prime}\left(x^{*}\right)^{-1}\left(F^{\prime}\left(y_{0}\right)-F^{\prime}\left(x^{*}\right)\right)\right|+\left|F^{\prime}\left(x^{*}\right)^{-1}\left(F^{\prime}\left(x_{0}\right)-F^{\prime}\left(x^{*}\right)\right)\right| \\
\leq & \frac{L\left|x_{0}-x^{*}\right|^{2}}{2\left(1-L_{0}\left|x_{0}-x^{*}\right|\right)}+\frac{3 L_{0}\left(\left|x_{0}-x^{*}\right|+\left|y_{0}-x^{*}\right|\right) M\left|x_{0}-x^{*}\right|}{4\left(1-g_{0}\left(\left|x_{0}-x^{*}\right|\right)\right)\left(1-L_{0}\left|x_{0}-x^{*}\right|\right)} \\
\leq & \frac{1}{2\left(1-L_{0}\left|x_{0}-x^{*}\right|\right)}\left[L+\frac{3 L_{0} M\left(1+g_{1}\left(\left|x_{0}-x^{*}\right|\right)\right)}{2\left(1-g_{0}\left(\left|x_{0}-x^{*}\right|\right)\right)}\right]\left|x_{0}-x^{*}\right| \\
= & g_{2}\left(\left|x_{0}-x^{*}\right|\right)\left|x_{0}-x^{*}\right|<g_{2}(r)\left|x_{0}-x^{*}\right|<\left|x_{0}-x^{*}\right|<r,
\end{aligned}
$$

which shows (2.17) for $n=0$ and $z_{0} \in U\left(x^{*}, r\right)$. Next, we need an estimate on $A_{0}^{-1}$. First, we have that

$$
\begin{aligned}
\frac{y_{0}-z_{0}}{y_{0}-x_{0}}= & \frac{-\frac{2}{3} F^{\prime}\left(x_{0}\right)^{-1} F\left(x_{0}\right)+\frac{1}{2} F^{\prime}\left(x_{0}\right)^{-1} F\left(x_{0}\right)}{-\frac{2}{3} F^{\prime}\left(x_{0}\right)^{-1} F\left(x_{0}\right)} \\
& +\frac{\frac{1}{2} F^{\prime}\left(x_{0}\right)^{-1} F\left(x_{0}\right) \frac{1}{1+\frac{3}{2}\left(\frac{F^{\prime}\left(x_{0}\right)}{F^{\prime}\left(y_{0}\right)}-1\right)}}{-\frac{2}{3} F^{\prime}\left(x_{0}\right)^{-1} F\left(x_{0}\right)} \\
= & -\frac{1}{4} B_{0}^{-1} F^{\prime}\left(x^{*}\right) F^{\prime}\left(x^{*}\right)^{-1}\left(2\left(F^{\prime}\left(x_{0}\right)-F^{\prime}\left(x^{*}\right)+F^{\prime}\left(x^{*}\right)\right)\right. \\
& +\frac{3}{2}\left(\left(F^{\prime}\left(y_{0}\right)-F^{\prime}\left(x^{*}\right)\right)+\left(F^{\prime}\left(x^{*}\right)-F^{\prime}\left(x_{0}\right)\right)\right),
\end{aligned}
$$

using (2.9), (2.16), (2.24), (2.26) and the definition of function $p$, we get that

$$
\begin{aligned}
\left|\frac{y_{0}-z_{0}}{y_{0}-x_{0}}\right| \leq & \frac{1}{4}\left|B_{0}^{-1} F^{\prime}\left(x^{*}\right)\right|\left[2\left(\left|F^{\prime}\left(x^{*}\right)^{-1}\left(F^{\prime}\left(x_{0}\right)-F^{\prime}\left(x^{*}\right)\right)\right|+\left|F^{\prime}\left(x^{*}\right)^{-1} F^{\prime}\left(x^{*}\right)\right|\right)\right. \\
& +\frac{3}{2}\left(\left|F^{\prime}\left(x^{*}\right)^{-1}\left(F^{\prime}\left(y_{0}\right)-F^{\prime}\left(x^{*}\right)\right)\right|+\left|F^{\prime}\left(x^{*}\right)^{-1}\left(F^{\prime}\left(x_{0}\right)-F^{\prime}\left(x^{*}\right)\right)\right|\right] \\
\leq & \frac{1}{4} \frac{2\left(1+L_{0}\left|x_{0}-x^{*}\right|\right)+\frac{3}{2} L_{0}\left(\left|x_{0}-x^{*}\right|+\left|y_{0}-x^{*}\right|\right)}{1-g_{0}\left(\left|x_{0}-x^{*}\right|\right)} \\
\leq & \frac{1}{4} \frac{2\left(1+L_{0}\left|x_{0}-x^{*}\right|\right)+\frac{3}{2} L_{0}\left(1+g_{1}\left(\left|x_{0}-x^{*}\right|\right)\right)\left|x_{0}-x^{*}\right|}{1-g_{0}\left(\left|x_{0}-x^{*}\right|\right)} \\
= & p\left(\left|x_{0}-x^{*}\right|\right) .
\end{aligned}
$$

Then, by definition of $A_{0}, p_{1},(2.9),(2.11),(2.16),(2.17)$ and (2.27) we get that

$$
\begin{aligned}
& \left|F^{\prime}\left(x^{*}\right)^{-1}\left(A_{0}-F^{\prime}\left(x^{*}\right)\right)\right| \\
& \leq 2\left|F^{\prime}\left(x^{*}\right)^{-1}\left(\left[x_{0}, z_{0}: F\right]-F^{\prime}\left(x^{*}\right)\right)\right| \\
& \quad+2\left|F^{\prime}\left(x^{*}\right)^{-1}\left(\left[x_{0}, z_{0}: F\right]-F^{\prime}\left(x^{*}\right)\right)\right|+\left|F^{\prime}\left(x^{*}\right)^{-1}\left(\left[x_{0}, z_{0}: F\right]-F^{\prime}\left(x^{*}\right)\right)\right| \\
& \quad+p\left(\left|x_{0}-x^{*}\right|\right)\left|F^{\prime}\left(x^{*}\right)^{-1}\left(\left[x_{0}, y_{0}: F\right]-F^{\prime}\left(x^{*}\right)\right)\right|+\left|F^{\prime}\left(x^{*}\right)^{-1}\left(F^{\prime}\left(x_{0}\right)-F^{\prime}\left(x^{*}\right)\right)\right| \\
& \leq 2\left(L_{1}\left|x_{0}-x^{*}\right|+L_{2}\left|y_{0}-x^{*}\right|\right)+2\left(L_{1}\left|x_{0}-x^{*}\right|+L_{2}\left|y_{0}-x^{*}\right|\right) \\
& \quad+L_{1}\left|x_{0}-x^{*}\right|+L_{2}\left|z_{0}-x^{*}\right|+p\left(\left|x_{0}-x^{*}\right|\right) \\
& \quad \times\left(L_{1}\left|x_{0}-x^{*}\right|+L_{2}\left|y_{0}-x^{*}\right|+L_{0}\left|x_{0}-x^{*}\right|\right) \\
& \leq\left(5 L_{1}+p\left(\left|x_{0}-x^{*}\right|\right)\left(L_{0}+L_{1}\right)\right)\left|x_{0}-x^{*}\right| \\
& \quad+\left(2 L_{2}+p\left(\left|x_{0}-x^{*}\right|\right) L_{2}\right)\left|y_{0}-x^{*}\right|+3 L_{2}\left|z_{0}-x^{*}\right| \\
& \leq \\
& \leq\left(5 L_{1}+p\left(\left|x_{0}-x^{*}\right|\right)\left(L_{0}+L_{1}\right)\right)\left|x_{0}-x^{*}\right|
\end{aligned}
$$




$$
\begin{aligned}
& +\left(2 L_{2}+L_{2} p\left(\left|x_{0}-x^{*}\right|\right)\right)\left|y_{0}-x^{*}\right| \\
& +3 L_{2}\left|z_{0}-x^{*}\right| \\
\leq & \left(5 L_{1}+p\left(\left|x_{0}-x^{*}\right|\right)\left(L_{0}+L\right)\right)\left|x_{0}-x^{*}\right| \\
& +\left(2 L_{2}+L_{2} p\left(\left|x_{0}-x^{*}\right|\right)\right) g_{1}\left(\left|x_{0}-x^{*}\right|\right)\left|x_{0}-x^{*}\right| \\
& +3 L_{2} g_{2}\left(\left|x_{0}-x^{*}\right|\right)\left|x_{0}-x^{*}\right| \\
= & p_{1}\left(\left|x_{0}-x^{*}\right|\right) .
\end{aligned}
$$

However, if we use instead of (2.11) the estimate (2.12) and (2.13), we obtain from (2.22) that

$$
\begin{aligned}
&\left|F^{\prime}\left(x^{*}\right)^{-1}\left(A_{0}-F^{\prime}\left(x^{*}\right)\right)\right| \\
& \leq 2\left|F^{\prime}\left(x^{*}\right)^{-1}\left(\left[x_{0}, z_{0}: F\right]-\left[x_{0}, y_{0}: F\right]\right)\right| \\
&+\left|F^{\prime}\left(x^{*}\right)^{-1}\left(\left[y_{0}, z_{0}: F\right]-F^{\prime}\left(x^{*}\right)\right)\right| \\
&+p\left(\left|x_{0}-x^{*}\right|\right)\left|F^{\prime}\left(x^{*}\right)^{-1}\left(\left[x_{0}, y_{0}: F\right]-F^{\prime}\left(x_{0}\right)\right)\right| \\
& \leq\left.2 L_{3}\left|z_{0}-x_{0}\right|+L_{1}\left|x_{0}-x^{*}\right|+L_{2}\left|z_{0}-x^{*}\right|\right)+p\left(\left|x_{0}-x^{*}\right|\right) L_{4}\left|y_{0}-x_{0}\right| \\
& \leq 2 L_{3}\left(\left|z_{0}-x^{*}\right|+\left|y_{0}-x^{*}\right|\right)+L_{1}\left|x_{0}-x^{*}\right| \\
&+L_{2}\left|z_{0}-x^{*}\right|+L_{4} p\left(\left|x_{0}-x^{*}\right|\right)\left(\left|y_{0}-x^{*}\right|+\left|x_{0}-x^{*}\right|\right) \\
& \leq\left(L_{1}+L_{4} p\left(\left|x_{0}-x^{*}\right|\right)\right)\left|x_{0}-x^{*}\right|+\left(2 L_{3}+L_{4} p\left(\left|x_{0}-x^{*}\right|\right)\right)\left|y_{0}-x^{*}\right| \\
&+\left(L_{2}+2 L_{3}\right)\left|z_{0}-x^{*}\right| \\
& \leq\left(L_{1}+L_{4} p\left(\left|x_{0}-x^{*}\right|\right)\right)\left|x_{0}-x^{*}\right| \\
&+\left(2 L_{3}+L_{4} p\left(\left|x_{0}-x^{*}\right|\right)\right) g_{1}\left(\left|x_{0}-x^{*}\right|\right)\left|x_{0}-x^{*}\right| \\
&+\left(L_{2}+2 L_{3}\right) g_{2}\left(\left|x_{0}-x^{*}\right|\right)\left|x_{0}-x^{*}\right| \\
&= p_{2}\left(\left|x_{0}-x^{*}\right|\right) .
\end{aligned}
$$

Then, from (2.2), (2.4), (2.28), (2.29) and the definition of function $p_{0}$ we get that $A_{0}$ is invertible and

$$
\left|A_{0}^{-1} F^{\prime}\left(x^{*}\right)\right| \leq \frac{1}{1-p_{0}(t)} .
$$

Then, using the last substep of method (1.2) for $n=0,(2.2),(2.7),(2.14),(2.18),(2.20)$ and (2.30) we obtain that

$$
\begin{aligned}
\left|x_{1}-x^{*}\right| & \leq\left|z_{0}-x^{*}\right|+\left|A_{0}^{-1} F\left(x^{*}\right)\right|\left|F^{\prime}\left(x^{*}\right)^{-1} F\left(z_{0}\right)\right| \\
& \leq\left[1+\frac{M}{1-p_{0}\left(\left|x_{0}-x^{*}\right|\right)}\right]\left|z_{0}-x^{*}\right| \\
& \leq\left[1+\frac{M}{1-p_{0}\left(\left|x_{0}-x^{*}\right|\right)}\right] g_{2}\left(\left|x_{0}-x^{*}\right|\right)\left|x_{0}-x^{*}\right| \\
& =g_{3}\left(\left|x_{0}-x^{*}\right|\right)\left|x_{0}-x^{*}\right|<g_{3}(r)\left|x_{0}-x^{*}\right|<\left|x_{0}-x^{*}\right|<r,
\end{aligned}
$$

which shows (2.18) for $n=0$ and $x_{1} \in U\left(x^{*}, r\right)$. By simply replacing $x_{0}, y_{0}, x_{1}$ by $x_{k}, y_{k}, x_{k+1}$ in the preceding estimates we arrive at estimates (2.16)-(2.18). Using the estimate $\left|x_{k+1}-x^{*}\right|<\left|x_{k}-x^{*}\right|<r$, we deduce that $x_{k+1} \in U\left(x^{*}, r\right)$ and $\lim _{k \rightarrow \infty} x_{k}=x^{*}$. To show the uniqueness part, let $Q=\int_{0}^{1} F^{\prime}\left(y^{*}+\theta\left(x^{*}-y^{*}\right) d \theta\right.$ for some $y^{*} \in \bar{U}\left(x^{*}, T\right)$ with $F\left(y^{*}\right)=0$. Using (2.6) we get that

$$
\begin{aligned}
\left|F^{\prime}\left(x^{*}\right)^{-1}\left(Q-F^{\prime}\left(x^{*}\right)\right)\right| & \leq \int_{0}^{1} L_{0}\left|y^{*}+\theta\left(x^{*}-y^{*}\right)-x^{*}\right| d \theta \\
& \leq \int_{0}^{1}(1-\theta)\left|x^{*}-y^{*}\right| d \theta \leq \frac{L_{0}}{2} R<1 .
\end{aligned}
$$

It follows from (2.20) and the Banach Lemma on invertible functions that $Q$ is invertible. Finally, from the identity $0=F\left(x^{*}\right)-F\left(y^{*}\right)=Q\left(x^{*}-y^{*}\right)$, we deduce that $x^{*}=y^{*}$. 
Remark 2.2 1. In view of (2.9) and the estimate

$$
\begin{aligned}
\left|F^{\prime}\left(x^{*}\right)^{-1} F^{\prime}(x)\right| & =\left|F^{\prime}\left(x^{*}\right)^{-1}\left(F^{\prime}(x)-F^{\prime}\left(x^{*}\right)\right)+I\right| \\
& \leq 1+\left|F^{\prime}\left(x^{*}\right)^{-1}\left(F^{\prime}(x)-F^{\prime}\left(x^{*}\right)\right)\right| \leq 1+L_{0}\left|x-x^{*}\right|
\end{aligned}
$$

condition (2.14) can be dropped and $M$ can be replaced by

$$
M(t)=1+L_{0} t .
$$

2. The results obtained here can be used for operators $F$ satisfying autonomous differential equations [3] of the form

$$
F^{\prime}(x)=P(F(x))
$$

where $P$ is a continuous operator. Then, since $F^{\prime}\left(x^{*}\right)=P\left(F\left(x^{*}\right)\right)=P(0)$, we can apply the results without actually knowing $x^{*}$. For example, let $F(x)=e^{x}-1$. Then, we can choose: $P(x)=x+1$.

3 . The radius $r_{A}$ given by (2.1) was shown by us to be the convergence radius of Newton's method [2-4]

$$
x_{n+1}=x_{n}-F^{\prime}\left(x_{n}\right)^{-1} F\left(x_{n}\right) \text { for each } n=0,1,2, \ldots
$$

under the conditions (2.9) and (2.10). It follows from (2.2) and $r<r_{A}$ that the convergence radius $r$ of the method (1.2) cannot be larger than the convergence radius $r_{A}$ of the second-order Newton's method (2.32). As already noted in [2,3] $r_{A}$ is at least as large as the convergence ball given by Rheinboldt [24]

$$
r_{R}=\frac{2}{3 L} \text {. }
$$

In particular, for $L_{0}<L$ we have that

$$
r_{R}<r
$$

and

$$
\frac{r_{R}}{r_{A}} \rightarrow \frac{1}{3} \text { as } \frac{L_{0}}{L} \rightarrow 0 .
$$

That is our convergence ball $r_{A}$ is at most three times larger than Rheinboldt's. The same value for $r_{R}$ was given by Traub [25].

4. It is worth noticing that method (1.2) is not changing when we use the conditions of Theorem 2.1 instead of the stronger conditions used in [1,2,8-23,25-27]. Moreover, we can compute the computational order of convergence (COC) defined by

$$
\xi=\ln \left(\frac{\left|x_{n+1}-x^{*}\right|}{\left|x_{n}-x^{*}\right|}\right) / \ln \left(\frac{\left|x_{n}-x^{*}\right|}{\left|x_{n-1}-x^{*}\right|}\right)
$$

or the approximate computational order of convergence

$$
\xi_{1}=\ln \left(\frac{\left|x_{n+1}-x_{n}\right|}{\left|x_{n}-x_{n-1}\right|}\right) / \ln \left(\frac{\left|x_{n}-x_{n-1}\right|}{\left|x_{n-1}-x_{n-2}\right|}\right) .
$$

This way we obtain in practice the order of convergence in a way that avoids the bounds involving estimates using estimates higher than the first Fréchet derivative of operator $F$.

\section{Numerical example}

We present a numerical example in this section.

Example 3.1 Let $D=[-\infty,+\infty]$. Define function $f$ of $D$ by

$$
f(x)=\sin (x) .
$$

Then we have for $x^{*}=0$ that $L_{0}=L=M=L_{3}=L_{4}=1, L_{1}=L_{2}=\frac{1}{2}$. The parameters are given in Table 1.

It is well known that due to errors and since higher order derivatives do not appear in the definition of $\xi$ or $\xi_{1}$ the computations may not necessarily lead to exactly $\xi_{1}=8$ as indicated by the Example 3.1. 
Table 1

$$
\begin{aligned}
& r_{A}=0.6667 \\
& r_{1}=0.4444 \\
& r_{2}=0.3361 \\
& r_{\overline{p_{1}}}=0.9240 \\
& r_{\overline{p_{2}}}=0.1506 \\
& r_{3}=0.0082 \\
& \xi_{1}=7.9389
\end{aligned}
$$

\section{Conclusion}

We presented a new local convergence analysis for an eighth-order method for solving equations based on contractive techniques and Lipschitz constants under hypotheses only on the first derivative. This way we expanded the applicability of method (1.2), since its convergence was shown using hypotheses up to the seventh derivative $[8,20]$. Moreover, we provided computable radius of convergence as well as error estimates not given in earlier studies $[8,20]$. The same advantages can be obtained if our technique is used on similar eighth-order methods listed in the references (see $[8,20]$ and the references therein).

Open Access This article is distributed under the terms of the Creative Commons Attribution 4.0 International License (http://creativecommons.org/licenses/by/4.0/), which permits unrestricted use, distribution, and reproduction in any medium, provided you give appropriate credit to the original author(s) and the source, provide a link to the Creative Commons license, and indicate if changes were made.

\section{References}

1. Amat, S.; Hernández, M.A.; Romero, N.: A modified Chebyshev's iterative method with at least sixth order of convergence. Appl. Math. Comput. 206(1), 164-174 (2008)

2. Amat, S.; Busquier, S.; Plaza, S.: Dynamics of the King's and Jarratt iterations. Aequationes. Math. 69, $212-213$ (2005)

3. Argyros, I.K.: Convergence and Application of Newton-type Iterations. Springer, Berlin (2008)

4. Argyros, I.K.; Hilout, Said.: Computational Methods in Nonlinear Analysis. World Scientific Publ. Co., New Jersey (2013)

5. Argyros, I.K.; Chen, D.; Quian, Q.: The Jarratt method in Banach space setting. J. Comput. Appl. Math. 51, 103-106 (1994)

6. Candela, V.; Marquina, A.: Recurrence relations for rational cubic methods I: the Halley method. Computing 44, 169-184 (1990)

7. Chen, J.: Some new iterative methods with three-order convergence. Appl. Math. Comput. 181, 1519-1522 (2006)

8. Chun, C.; Neta, B.; Scott, M.: Basins of attraction for optimal eighth order methods to find simple roots of nonlinear equations. Appl. Math. Comput. 227, 567-592 (2014)

9. Cordero, A.; Torregrosa, J.: Variants of Newton's method using fifth order quadrature formulas. Appl. Math. Comput. 190, 686-698 (2007)

10. Cordero, A.; Maimo, J.; Torregrosa, J.; Vassileva, M.P.; Vindel, P.: Chaos in King's iterative family. Appl. Math. Lett. 26, 842-848 (2013)

11. Cordero, A.; Magrenan, A.; Quemada, C.; Torregrosa, J.R.: Stability study of eight-order iterative methods for solving nonlinear equations. J. Comput. Appl. Math. (to appear)

12. Ezquerro, J.A.; Hernández, M.A.: A uniparametric Halley-type iteration with free second derivative. Int. J. Pure Appl. Math. 6(1), 99-110 (2003)

13. Ezquerro, J.A.; Hernández, M.A.: New iterations of R-order four with reduced computational cost. BIT Numer. Math. 49, 325-342 (2009)

14. Frontini, M.; Sormani, E.: Some variants of Newton's method with third order convergence. Appl. Math. Comput. 140, 419-426 (2003)

15. Gutiérrez, J.M.; Hernández, M.A.: Recurrence relations for the super-Halley method. Comput. Math. Appl. 36(7), 1-8 (1998)

16. Hernández, M.A.; Salanova, M.A.: Sufficient conditions for semilocal convergence of a fourth order multipoint iterative method for solving equations in Banach spaces. Southwest J. Pure Appl. Math. (1), 29-40 (1999)

17. Kanwar, M.V.; Kukreja, V.K.; Singh, S.: On some third-order iterative methods for solving nonlinear equations. Appl. Math. Comput. 171, 272-280 (2005)

18. Kou, J.; Li, Y.: An improvement of the Jarratt method. Appl. Math. Comput. 189, 1816-1821 (2007)

19. Parhi, S.K.; Gupta, D.K.: Semilocal convergence of a Stirling-like method in Banach spaces. Int. J. Comput. Methods 7(02), 215-228 (2010)

20. Petkovic, M.S.; Neta, B.; Petkovic, L.; Džunič, J.: Multipoint Methods for Solving Nonlinear Equations. Elsevier, Amsterdam (2013)

21. Potra, F.A.; Ptak, V.: Nondiscrete induction and iterative processes. Research Notes in Mathematics, vol. 103. Pitman Publ., Boston (1984)

22. Rall, L.B.: Computational Solution of Nonlinear Operator Equations. Robert E. Krieger, New York (1979) 
23. Ren, H.; Wu, Q.; Bi, W.: New variants of Jarratt method with sixth-order convergence. Numer. Algorithms 52(4), 585-603 (2009)

24. Rheinboldt, W.C.: An adaptive continuation process for solving systems of nonlinear equations. In: Tikhonov, A.N.; et al. (eds.) Mathematical models and numerical methods, pub. 3, (19), pp. 129-142. Banach Center, Warsaw (1975)

25. Traub, J.F.: Iterative Methods for the Solution of Equations. Prentice Hall, Englewood Cliffs (1964)

26. Weerakoon, S.; Fernando, T.G.I.: A variant of Newton's method with accelerated third-order convergence. Appl. Math. Lett. 13, 87-93 (2000)

27. Wang, X.; Kou, J.: Convergence for modified Halley-like methods with less computation of inversion. J. Differ. Equ. Appl. 19(9), 1483-1500 (2013) 